\title{
Pressure induced lattice expansion and phonon softening in layered $\operatorname{ReS}_{2}$
}

Cite as: J. Appl. Phys. 128, 085904 (2020); https://doi.org/10.1063/5.0014347

Submitted: 19 May 2020 . Accepted: 15 August 2020 . Published Online: 31 August 2020

(D) Pinku Saha, Bishnupada Ghosh, Aritra Mazumder, Konstantin Glazyrin, and (D) Goutam Dev Mukherjee

\section{COLLECTIONS}

Paper published as part of the special topic on Beyond Graphene: Low Symmetry and Anisotropic 2D Materials BGLS2020
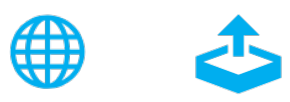

\section{ARTICLES YOU MAY BE INTERESTED IN}

Beyond Graphene: Low-Symmetry and Anisotropic 2D Materials

Journal of Applied Physics 128, 140401 (2020); https://doi.org/10.1063/5.0030751

A practical guide for crystal growth of van der Waals layered materials

Journal of Applied Physics 128, 051101 (2020); https://doi.org/10.1063/5.0015971

The optical signature of few-layer $\mathrm{ReSe}_{2}$

Journal of Applied Physics 128, 044302 (2020); https://doi.org/10.1063/5.0015289

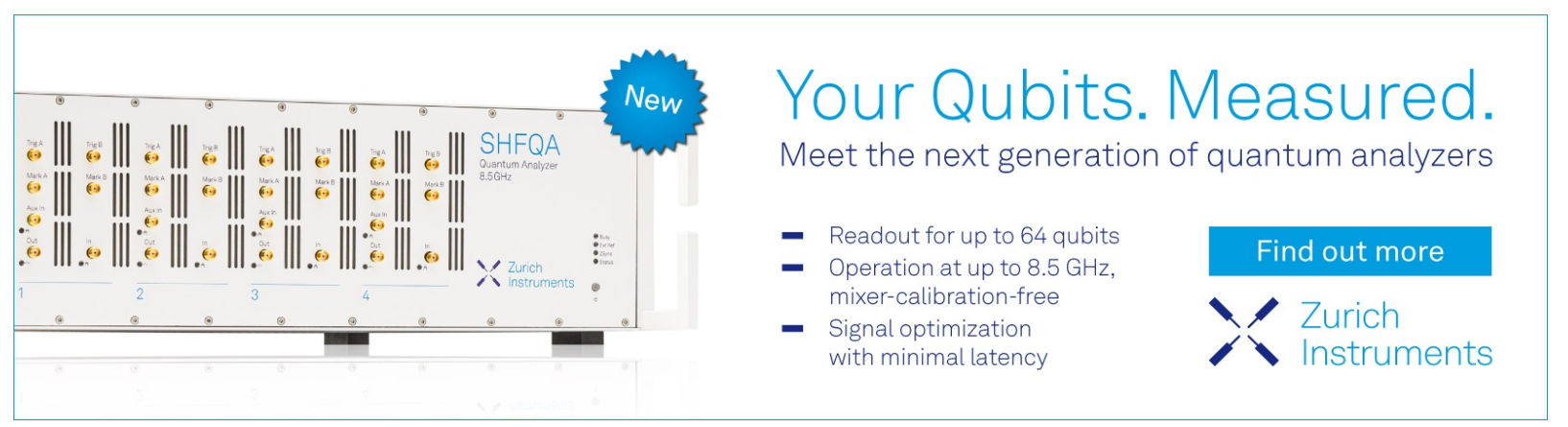




\title{
Pressure induced lattice expansion and phonon softening in layered $\mathrm{ReS}_{2}$
}

\author{
Cite as: J. Appl. Phys. 128, 085904 (2020); doi: 10.1063/5.0014347 \\ Submitted: 19 May 2020 - Accepted: 15 August 2020 . \\ Published Online: 31 August 2020
}

Pinku Saha, ${ }^{7}$ (D) Bishnupada Ghosh, ${ }^{7}$ Aritra Mazumder, ${ }^{7}$ Konstantin Glazyrin, ${ }^{2}$ and Goutam Dev Mukherjee ${ }^{1, a)}$ (D)

\begin{abstract}
AFFILIATIONS
${ }^{1}$ National Centre for High Pressure Studies, Department of Physical Sciences, Indian Institute of Science Education and Research Kolkata, Mohanpur Campus, Mohanpur, 741246 Nadia, West Bengal, India

${ }^{2}$ Photon Science, Deutsches Elektronen Synchrotron, 22607 Hamburg, Germany
\end{abstract}

Note: This paper is part of the Special Topic on Beyond Graphene: Low Symmetry and Anisotropic 2D Materials.

${ }^{a}$ Author to whom correspondence should be addressed: goutamdev@iiserkol.ac.in

\begin{abstract}
We report high pressure $\mathrm{x}$-ray diffraction and systematic Raman measurements on a $\mathrm{ReS}_{2}$ sample, which is mechanically exfoliated from a single crystal. A few new Bragg peaks are observed to emerge above $6 \mathrm{GPa}$ indicating a structural transition from distorted 1T to distorted $1 \mathrm{~T}^{\prime}$ in a triclinic structure. The same is corroborated by the appearance of new Raman modes in the same pressure range. Softening of the Raman modes corresponding to Re atom vibrations is observed in the distorted $1 \mathrm{~T}^{\prime}$ phase in the pressure range of $15-25 \mathrm{GPa}$. In the same pressure range, the anomalous change in the volume is found to be induced by the lattice expansion. The volume expansion is related to the sliding of layers leading to octahedral distortion and an increase in octahedral volume. The sample is found to be very incompressible above $25 \mathrm{GPa}$ with respect to below $15 \mathrm{GPa}$ data. The same is also reflected in the Raman mode shifts with pressure.
\end{abstract}

Published under license by AIP Publishing. https://doi.org/10.1063/5.0014347

\section{INTRODUCTION}

In recent times, transition metal dichalcogenides (TMDs) have been shown to be promising objects for exploring quantum phenomena due to their rich structural, electronic, and optical properties. ${ }^{1-6}$ Under pressure, layered two-dimensional (2D) forms of these materials exhibit very interesting properties like superconductivity ${ }^{7,8}$ and charge density waves. ${ }^{9,10}$ TMDs are found to adopt different crystal structures. ${ }^{11-14}$ Among the different structures, the most common stable phase is the hexagonal $(\mathrm{H})$ phase, while the metastable triclinic (T) phase is also observed to be stable in few cases. In the $\mathrm{H}$ phase, the metal atoms sit at prismatic positions, whereas in the $\mathrm{T}$ phase, they occupy the octahedral positions between two chalcogen layers. Among TMD-trilayers, a weak van der Waals force is present. In $\mathrm{ReS}_{2}$, this force is reported to be very small, less than $8 \%$ with respect to that of $\mathrm{MoS}_{2}$, and is caused by the Peierls distortion of the low triclinic symmetry of $\mathrm{ReS}_{2}{ }^{7,15-19}$ This weak coupling makes bulk $\mathrm{ReS}_{2}$ exhibit monolayer behavior. ${ }^{7,20}$

The effect of high pressures in the hexagonal phase of TMDs has been extensively studied. ${ }^{3,21-26}$ An electronic transition from a semiconducting to a metallic phase is reported during isostructural transition $\left(2 \mathrm{H}_{c}-2 \mathrm{H}_{a}\right)$ at high pressures for powder samples. ${ }^{3,21-24}$ In contrast, in exfoliated samples, the same electronic transition is reported during strain induced structural transition from the hexagonal to the triclinic phase at high pressure. ${ }^{25,26}$ Due to very weak van der Waals interlayer interaction in distorted $1 \mathrm{~T}, \mathrm{ReS}_{2}$ application of high pressure can reduce the interlayer distances rapidly and, hence, can change material properties easily. ${ }^{7,17-20,27,28}$ A few high pressure XRD studies on powder samples show structural transition from the distorted $1 \mathrm{~T}$ to $1 \mathrm{~T}^{\prime}$ phase in the pressure range of $7.7-11 \mathrm{GPa} \cdot{ }^{18,27,28}$ As these two phases differ by only layer stacking order, experimental distinction by Raman study is very difficult. ${ }^{19}$ From the inflection points in the ratio of Raman mode intensities and change in the slope of Raman mode values with pressure in combination with first principles calculation, an intralayer transition followed by an interlayer transition in the range of $8-19.6 \mathrm{GPa}$ is reported by Yan et al. ${ }^{17}$ A very recent first principle study by Sheremetyeva et al. on $\mathrm{ReS}_{2}$ under pressure reported two different slopes for pressure variation of Raman mode frequencies for $1 \mathrm{~T}$ and $1 \mathrm{~T}^{\prime}$ phases, respectively. ${ }^{19}$

All the above results indeed require systematic high pressure Raman and XRD measurements to correlate the structural and 
vibrational properties. In our work, we have carried out a detailed systematic high pressure investigation on exfoliated fewmicrometers thick $\mathrm{ReS}_{2}$ using Raman spectroscopy and XRD measurements up to about $45 \mathrm{GPa}$ using a diamond anvil cell (DAC). Analysis of XRD data shows a structural transition above $6 \mathrm{GPa}$, followed by a lattice expansion of the new structure in the pressure range of $15-25 \mathrm{GPa}$. In the same pressure range, softening in the Raman modes corresponding to the Re atoms are observed.

\section{EXPERIMENTAL}

High quality layered single crystal of distorted $1 \mathrm{~T}-\mathrm{ReS}_{2}$ is purchased from HQ-graphene. Sample is mechanically exfoliated for the ambient and high pressure studies. We have used a pistoncylinder type DAC from EasyLab Co. (UK) for our high pressure Raman spectroscopy measurements. Exfoliated samples are placed on the lower diamond anvil of culet $300 \mu \mathrm{m}$ for the collection of ambient Raman spectra. A T-301 steel gasket having a central hole of $100 \mu \mathrm{m}$, which is preindented to a thickness of $50 \mu \mathrm{m}$ is used for high pressure measurements. Three different types of pressure transmitting media (PTM): mixture of methanol-ethanol at a ratio 4:1, iso-propanol, and silicone oil are utilized for high pressure Raman measurements. For the determination of pressure, few small ruby chips (approximate size $3-5 \mu \mathrm{m}$ ) are loaded along with the samples and pressure is measured from the shift of Ruby fluorescence peak. ${ }^{29}$ Raman spectra are collected in the back scattering geometry using a confocal micro-Raman system (Monovista from SI $\mathrm{GmbH}$ ) using $1500 \mathrm{~g} / \mathrm{mm}$ grating with a spectral resolution better than $1.2 \mathrm{~cm}^{-1}$ using appropriate edge filter with cutoff around $50 \mathrm{~cm}^{-1}$. Sample is excited using sapphire SF optically pumped semiconductor laser of wavelength $488 \mathrm{~nm}$. Raman spectra are collected with a $50 \times$ microscope objective lens (infinitely corrected) having laser spot size about $2 \mu \mathrm{m}$. Laser power is kept constant to a maximum $15 \mathrm{~mW}$ to avoid the heating of the sample.

$\mathrm{X}$-ray diffraction measurements under ambient conditions as well as at high pressures are carried out in the Petra-III P02.2 beamline using a monochromatic $\mathrm{x}$ ray having wavelength of $0.2907 \AA$. A very narrow beam of $\mathrm{x}$ ray around $1.2 \times 2.3 \mu \mathrm{m}^{2}$ is chosen for the diffraction measurements. For high pressure XRD measurements, the exfoliated samples are loaded in the symmetric anvil DAC with culet flat $150 \mu \mathrm{m}$. In this case, neon is used as pressure transmitting medium. Small amount of gold powder is mixed with the sample, which acts as pressure marker. ${ }^{30}$ Sample to detector distance, tilt angle are calibrated using $\mathrm{CeO}_{2}$ standard. Collected two-dimensional diffraction images are integrated to intensity vs $2 \theta$ profile using Dioptas software ${ }^{31}$ and then analyzed using the CRYSFIRE ${ }^{32}$ and Rietveld fitting program of GSAS. ${ }^{33}$

\section{RESULTS AND DISCUSSION}

\section{A. Characterization of the ambient sample}

For characterization of the crystal structure, we have recorded the XRD pattern of the sample under ambient conditions. The pattern is indexed to a distorted layered structure of triclinic symmetry with space group $P \overline{1}$. Lattice parameters of this phase are found to be $a=6.581(7), \quad b=6.389(8)$, and $c=6.443(7) \AA$; $\alpha=118.9(3)^{\circ}, \quad \beta=94.1(2)^{\circ}$, and $\gamma=106.4(4)^{\circ}$ with volume
$\left(V_{0}\right)=220.34(5) \AA^{3}$ and has an excellent agreement with the 1T-phase reported in literature studies. ${ }^{18,27,34,35}$ The XRD pattern along with its Rietveld refinement is shown in Fig. 1. Rietveld refinement is carried out using the atom positions given in the literature as the starting model. ${ }^{34,35}$ We could achieve a best Rietveld refinement of the ambient XRD pattern of $\mathrm{ReS}_{2}$ with a $9 \% R_{w p}$ value. In a unit cell, each rhenium atom is surrounded by six sulfur atoms, occupying an octahedral coordination as shown in Fig. S1 (a) in the supplementary material. $\mathrm{ReS}_{6}$ octahedra are packed together such that mono-layer of $\mathrm{ReS}_{2}$ is stacked along the $a$-axis [Figs. S1(b) and S1(c) in the supplementary material]. In a unit cell, there are four Re atoms and they are arranged in a zigzag way as seen from Fig. S1(c) in the supplementary material due to the Peierls distortion, ${ }^{7,28}$ which restricts the ordered stacking.

As per space group symmetry, each unit cell consists of 12 atoms corresponding to 36 phonon modes at the center of the Brillouin zone. Out of 36 phonon modes, 18 are Raman active. ${ }^{16,36}$ In Fig. 2, we have shown the Raman spectrum in the spectral range of $100-450 \mathrm{~cm}^{-1}$ of the sample placed on the lower diamond anvil culet at ambient conditions. We have observed all 18 vibrational modes, and they are listed at the right side of Fig. 2. All the mode values match very well with those reported in literature studies. $^{7,17,18,36,37}$ Modes below $250 \mathrm{~cm}^{-1}$ can be attributed to the vibrations of heavy Re atoms and those above $250 \mathrm{~cm}^{-1}$ to the vibrations of the relatively light element, S-atoms in the unit cell. $A_{1 g}, E_{g}$, and $C_{p}$ modes are related to the out-off-plane, in-plane, and coupled vibrations of Re and $\mathrm{S}$ atoms, respectively. The positions of $A_{1 g}-1$, $E_{g}-1$, and $E_{g}-3$ and the difference between $E_{g}-1$ and $A_{1 g}-1$ modes are dependent on the number of layers as per the analysis of unpolarized Raman spectra reported by Chenet et al. ${ }^{37}$ They observe that $A_{1 g}-1$ mode frequency increases non-linearly from the initial value of $133.1 \pm 0.1 \mathrm{~cm}^{-1}$ corresponding to single layer upon

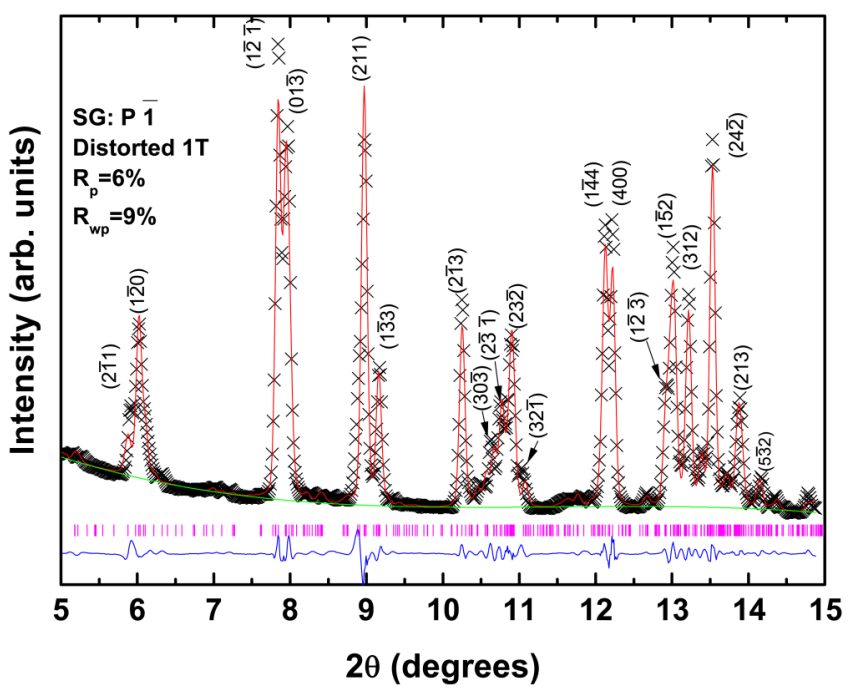

FIG. 1. Rietveld refinement of the ambient x-ray diffraction pattern of $\operatorname{ReS}_{2}$. Crosses: experimental data; red line: Rietveld fit; green line: background fit; blue line: residual; and magenta bars: Bragg peak positions. 


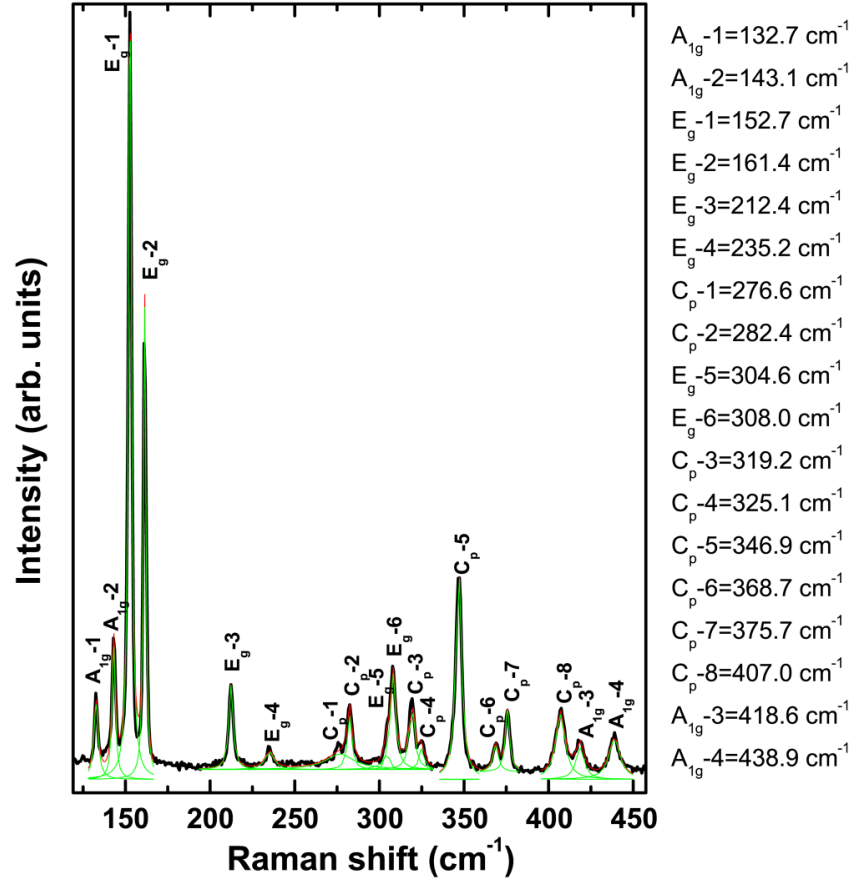

FIG. 2. Raman spectrum of distorted $1 \mathrm{~T} \mathrm{ReS}_{2}$ placed on the lower diamond anvil. All the mode values are indicated in the vertical list.

increase of the layer number, whereas $E_{g}-1$ and $E_{g}-3$ mode frequencies decrease linearly. ${ }^{37}$ We find $A_{1 g}-1$ mode at $132.7 \mathrm{~cm}^{-1}$, which is very close to the value for the single layer as reported by the above study. The difference of $E_{g}-1$ and $A_{1 g}-1$ modes is reported to decrease with the number of layers non-linearly. ${ }^{37}$ In our study, this difference is found to be $20 \mathrm{~cm}^{-1}$, which is larger than the single layer value $16.8 \pm 0.2 \mathrm{~cm}^{-1}$ reported by Chenet et al. ${ }^{37}$ Other studies on the layer dependence of Raman spectra $^{38,39}$ show that the difference increases with the number of layers from $16.7 \mathrm{~cm}^{-1}$ and has a value $19.9 \mathrm{~cm}^{-1}$ for four layer configuration. The ratio of the intensities between the $E_{1 g}-1$ and $E_{1 g}-2$ modes is found to be around 1.7 using $488 \mathrm{~nm}$ laser excitation, which is close to one to two layers as per McCreary et al. ${ }^{40}$ It is also reported that bulk $\mathrm{ReS}_{2}$ behaves like a mono-layer due to vibrational decoupling. ${ }^{7}$ Due to the above discrepancies in the layer number identification using Raman spectra analysis, we shall identify our exfoliated sample as a exfoliated few-micrometer thick layered sample.

\section{B. High pressure studies}

Pressure evolution of the x-ray diffraction patterns at selected pressure points at room temperature is shown in Fig. 3. Above $6.1 \mathrm{GPa}$, a few new diffraction lines are found to appear around $2 \theta=8^{\circ}$. From this figure, it is evident that new diffraction lines appear as pressure increases, which are marked by black arrows. In contrast to the observation of a new single XRD peak as reported by Hou et al. and Wang et al., we have observed three to four new diffraction lines as pressure increases. ${ }^{18,27}$ All the diffraction patterns

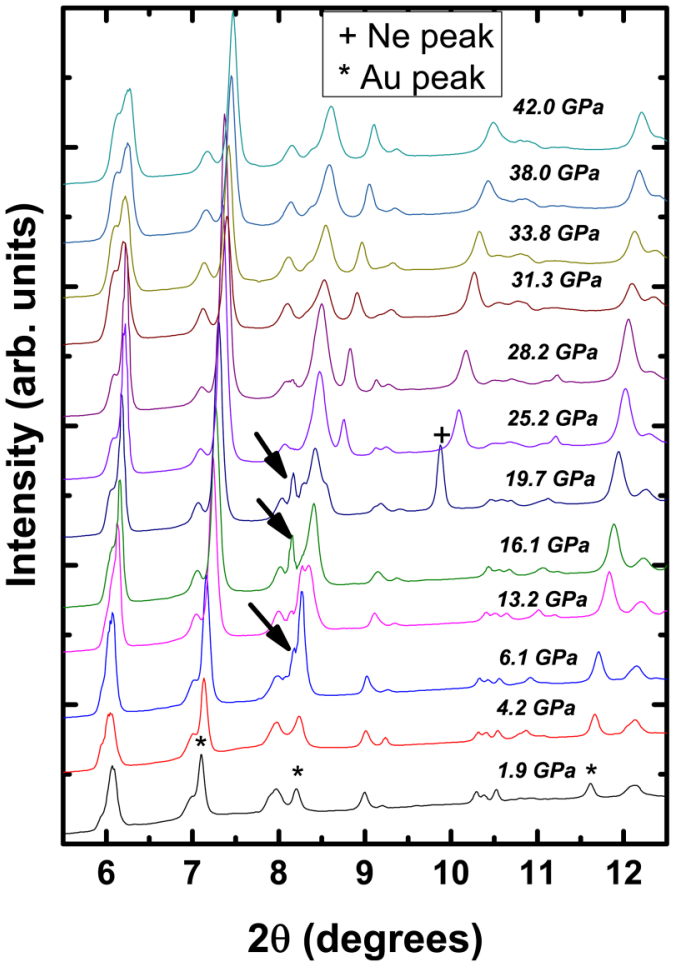

FIG. 3. The evolution of the x-ray diffraction patterns at selected pressure points. Emergence of new peaks are marked by arrows.

above $6.1 \mathrm{GPa}$ are indexed to a different set of lattice parameters in the same triclinic structure with space group $P \overline{1}$ indicating an iso-structural transition. In Fig. S2 in the supplementary material, we have shown the Rietveld refinement fit of the new structure at 13.2 GPa. Mismatch in the intensity profile in few Bragg peaks in Rietveld fitting may be due to the effect of preferred orientation of the crystallites as $\mathrm{ReS}_{2}$ is exfoliated from a single crystal. The same structure is reported as distorted $1 \mathrm{~T}^{\prime}$ in literature studies. ${ }^{18,27,28} \mathrm{XRD}$ pattern at $6.1 \mathrm{GPa}$ could be indexed to the triclnic structure with lattice parameters: $a=6.243(5) \AA, b=6.641(7) \AA, c=6.465(7) \AA$, $\alpha=102.8(2)^{\circ}, \quad \beta=103.4(4)^{\circ}$, and $\gamma=124.0(3)^{\circ}$ with volume $(V)=194.46(4) \AA^{3}$ and are in excellent agreement with those of $1 T^{\prime}$ structure reported by Wang et al. ${ }^{18}$ Packing of the $\mathrm{ReS}_{6}$ octahedra in a unit cell are shown in Fig. S3(a) in the supplementary material. All the atoms are observed to occupy slightly different positions in the unit cell with respect to those reported by Wang et al. ${ }^{18}$ The atom positions of distorted $1 T$ and $1 T^{\prime}$ structures obtained from our study are compared with the $1 \mathrm{~T}^{\prime}$ structure at 20.1 GPa reported by above group ${ }^{18}$ in Table I in the supplementary material. In the distorted $1 T^{\prime}$ structure, sulfur atoms of adjacent layers are observed to penetrate inside the unit cell of mono-layer $\mathrm{ReS}_{2}$ to form a triple layer contribution in a unit cell [Fig. S3(b) in the supplementary material]. Mono-layers are formed in the $b c$-plane and are stacked in the direction of the $a$-axis, which is evident from this figure. Rhenium atoms are found to present a 
zigzag chain in similar fashion as observed in the previous low pressure structure [Fig. S3(c) in the supplementary material]. In the $1 \mathrm{~T}^{\prime}$ phase, we do not find any structural change till $42 \mathrm{GPa}$.

In Fig. 4(a), we have plotted the volume dependence with pressure up to $42 \mathrm{GPa}$. The volume of this structure is found to decrease upon increasing pressure up to about $16 \mathrm{GPa}$ and shows anomalous changes in the pressure range of $16-25 \mathrm{GPa}$. In this pressure range, a volume expansion is observed and then followed by the normal compression behavior above $25 \mathrm{GPa}$. Similar expansion in the volume under hydrostatic condition was reported in the layered metal-organic framework. ${ }^{41}$ In Fig. S4 in the supplementary material, we have shown the Rietveld refinement fit at $16.1 \mathrm{GPa}$ and 25.2 GPa. The fittings show excellent match to the XRD patterns and no further structural transitions. Pressure induced strain has a very important role in changing the structural and the electronic properties of materials. ${ }^{25,26,42-44}$ To see the response of strain in our sample, we have estimated Eulerian strain $\left(f_{E}\right)$ and corresponding normalized pressure $(H)$ using the following equations: ${ }^{42,45}$

$$
H=\frac{P}{3 f_{E}\left(1+2 f_{E}\right)^{5 / 2}}
$$

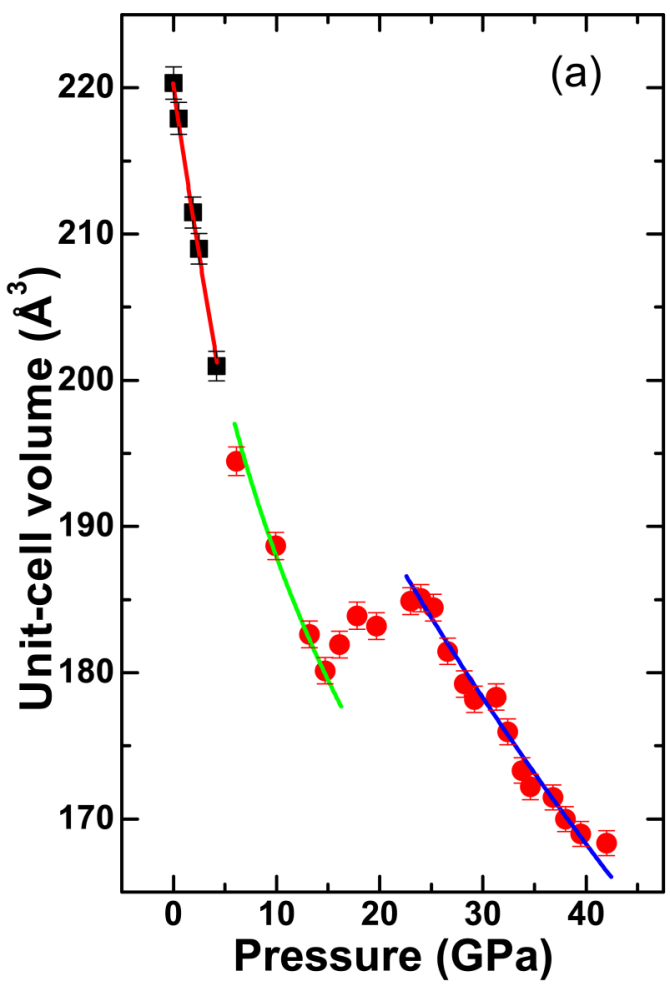

and

$$
f_{E}=\frac{1}{2}\left[\left(\frac{V_{0}}{V}\right)^{2 / 3}-1\right],
$$

where $V_{0}$ is the ambient pressure value of $220.34 \AA^{3} . H$ is linear with respect to $f_{E},{ }^{46,47}$

$$
H=K_{0}+\frac{3}{2} K_{0}\left(K^{\prime}-4\right) f_{E},
$$

where $V$ is the volume at pressure $P, K_{0}$ is the bulk modulus, and $K^{\prime}$ is the first derivative of bulk modulus. Eulerian strain vs normalized pressure is shown in Fig. 4(b). A jump in $H$ is observed above 6.1 GPa, where the iso-structural transition occurs. In the pressure range $16-25 \mathrm{GPa}, H$ is almost doubled, even though $f_{E}$ remains almost constant indicating a deformation of the unit cell to accommodate the applied pressure. Deformation of unit cell probably leads to expansion in volume in this pressure range. Above $25 \mathrm{GPa}$, the reduced pressure value is found to decrease with increasing $f_{E}$. We have performed three different linear fits separately: (i) in the pressure region ambient to $4.2 \mathrm{GPa}$ (belongs to the $1 \mathrm{~T}$-phase); (ii)

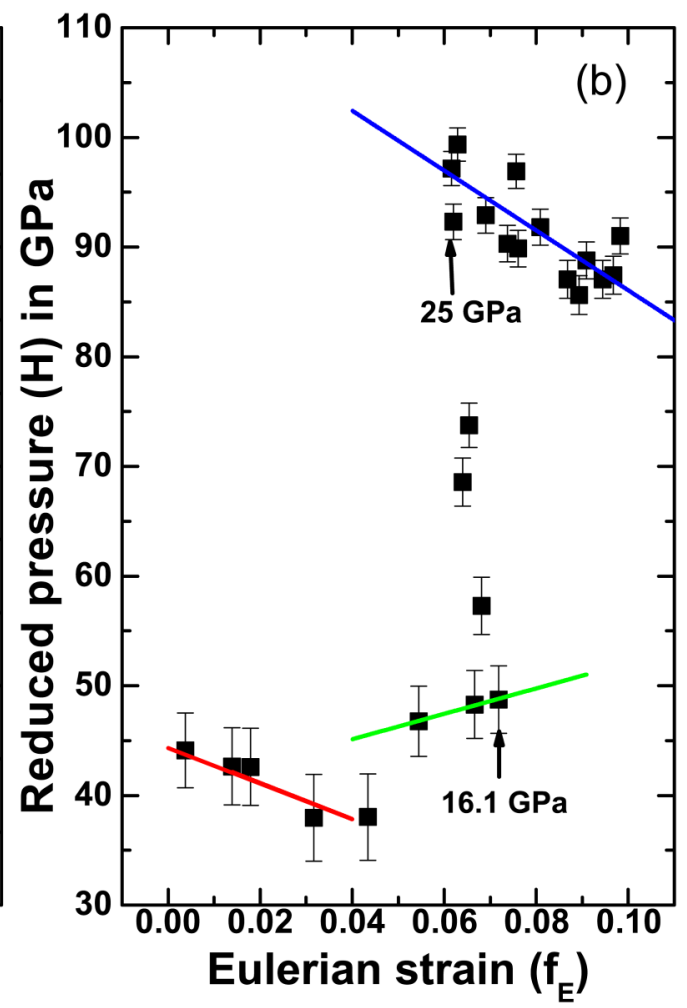

FIG. 4. (a) Evolution of volume as a function of pressure with EOS fitting (red line-1T, green line-region-I of $1 T^{\prime}$, and blue line-region-II of $1 T^{\prime}$ ). Black filled squares represent the volume of distorted $1 \mathrm{~T}$, and red filled circles represent the volume of distorted $1 \mathrm{~T}^{\prime}$ of $\operatorname{ReS}_{2}$. (b) Eulerian strain $\left(f_{E}\right)$ vs reduced pressure $(H)$ in the entire pressure range of our study. All solid lines (red line-the $1 \mathrm{~T}$ phase, green line-region-I of $1 \mathrm{~T}^{\prime}$, and blue line-region-ll of the $1 \mathrm{~T}^{\prime}$ phase) represent fit to Eq. (3), and unfitted portion is the volume expansion region. 
in the pressure region $6.1-16 \mathrm{GPa}$ (region- $\mathrm{I}$ in the $1 \mathrm{~T}^{\prime}$ phase), and (iii) in the pressure region $25-44 \mathrm{GPa}$ (region-II in the $1 \mathrm{~T}^{\prime}$ phase).

Fitting to the $1 \mathrm{~T}$ phase reveals $K_{0}=44(2) \mathrm{GPa}$ and $K^{\prime}=1.6(3)$, indicating almost linear and large compressibility behavior. Reported values of $K_{0}$ and $K^{\prime}$ for bulk powder samples in the literature are $23 \pm 4 \mathrm{GPa}$ and $29 \pm 8$, respectively, by Hou et al. ${ }^{27}$ and $35.6 \pm 5.2 \mathrm{GPa}$ and $10.8 \pm 2.4$, respectively, by Wang et al. ${ }^{18}$ The bulk modulus value in the present case is found to be high with respect to Hou et al., ${ }^{27}$ while it is observed to be close to Wang et al. within their error limit. ${ }^{18}$ The first group performed the experiment on the powder sample using an alcohol mixture as PTM using a $30 \times 30 \mu \mathrm{m}^{2} \mathrm{x}$-ray beam of wavelength of $0.4959 \AA$ and obtained $K_{0}$ and $K^{\prime}$ values by fitting P-V data to 3rd-order BM EOS considering four data points (ambient to 6.4 GPa). They measured pressure from the ruby scale. ${ }^{29}$ A small error in determination of pressure would result in a large deviation in volume, and their observed $K_{0}$ and $K^{\prime}$ values. Indeed, they reported $K_{0}$ to be $49 \pm$ $3 \mathrm{GPa}$ while taking second-order BM EOS. On the other hand, the other group carried out the experiment on a powder sample using neon as PTM using a $5 \mu \mathrm{m}$ diameter $\mathrm{x}$-ray beam of wavelength of $0.4066 \AA$ and determined $V_{0}, K_{0}$, and $K^{\prime}$ values by fitting the $\mathrm{P}-\mathrm{V}$ data to 3rd-order BM EOS considering eight data points (17.7 GPa). They also estimated $K_{0}$ and $K^{\prime}$ from the fitting of the $H$ vs $f_{E}$ plot, which is calculated taking $V_{0}$ from the analysis of BM EOS as they do not report ambient volume of the sample. They also measured pressure from ruby. In contrast to the above studies, pressure in our study is estimated from the analysis of the XRD pattern of $\mathrm{Au}$. XRD patterns of both the sample and Au are collected from a $2 \times 3 \mu \mathrm{m}^{2}$ area simultaneously that minimizes the error in the measured pressure values. We have carried out the experiment on a fewmicrometer thick exfoliated sample, while all other experimental conditions remain similar to that of Wang et al. ${ }^{18}$ We have determined $K_{0}$ and $K^{\prime}$ from the fitting of the $H$ vs $f_{E}$ curve taking $V_{0}$ from our study, and it produces relatively small error in the $K_{0}$ and $K^{\prime}$ values. Fitting of P-V data to 3 rd-order BM EOS yield $K_{0}=$ $43.9 \pm 7 \mathrm{GPa}$ and $K^{\prime}=1.4 \pm 0.2$ and are slightly higher than that of Wang et al. ${ }^{18}$ Both estimations following two different paths in this study are found to agree very well. So, one can speculate that different experimental conditions, determination of pressure using different methods, relatively small pressure range, and lack of perfect determination of sample volume may result in different values of bulk modulus and its derivative.

Fitting to the region-I of the $1 \mathrm{~T}^{\prime}$-phase provides $K_{0}=40(2) \mathrm{GPa}$ and $K^{\prime}=5.9(6)$, which shows a larger pressure dependence of bulk modulus in comparison to the $1 \mathrm{~T}$ phase. Interestingly above $25 \mathrm{GPa}$ in region-II, the fitting gives the values of $K_{0}=109(5) \mathrm{GPa}$ and $K^{\prime}=2.7(6)$, much larger bulk modulus in comparison to lower pressures. Similar bulk modulus values are also observed in other transition metal dichalcogenides in their triclinic phase. ${ }^{25,26}$ The fitting of the Eulerian strain vs normalized pressure in the study by Wang et al. taking $8.9 \mathrm{GPa}$ unit-cell volume as reference volume produced a bulk modulus of $90.1 \pm 2.2 \mathrm{GPa}$ and a pressure derivative of $5.1 \pm 0.3$, and the value of bulk modulus in their study is found to be very close to the present study. ${ }^{18}$ We have shown the EOS fitting in Fig. 4(a) taking the values of $K_{0}$ and $K^{\prime}$ from the fitting of the $f_{E} v s H$ plot and ambient sample volume in this present study as $V_{0}$. In Fig. S5 in the supplementary material, we have shown the evolution of lattice parameters in both the phases. In the low pressure phase, maximum compression is observed along the $a$-axis, the stacking direction of the mono-layers, as expected due to the weak van der Waal's interaction along that direction. Compression of the $c$ axis is observed to be high with respect to the $a$ axis in region-I of the $1 \mathrm{~T}^{\prime}$. Above $15 \mathrm{GPa}$, in the volume expansion region, the $c$-axis remains almost unchanged up to $25 \mathrm{GPa}$, while athe-axis increases slowly similar to volume. Above $25 \mathrm{GPa}$, in region-II, both axes show high compressibility with respect to the $b$-axis.

To understand the volume expansion behavior in the range of 16-25 GPa, we have plotted certain atom separations with pressure (Fig. 5). The distance between different sets of atoms (Re5-Re6, Re6-S2, Re5-S3, and Re6-S3) is measured in a single unit cell [Figs. 5(a) and 5(b)], while between S2 and S3 is measured considering sulfur atom from adjacent layers. From the top left corner of Fig. 5(a), it is evident that S2 is of different mono-layer and S2-S3 distance is between side by side S2 and S3 atoms from adjacent layers. All the distances show similar behavior, decrease up to $14.7 \mathrm{GPa}$, then suddenly change their trend and increase up to $25 \mathrm{GPa}$, and followed by a decrease till $42 \mathrm{GPa}$. It is expected that an increment in the distance among Re6 and Re5, S2, and S3 atoms in the unit cell in pressure range of 16-25 GPa would result in a decrease in the distance between S2 and S3 atoms of the adjacent layers. Rather, we find that it also increases in the same pressure range. It is only possible in the case of presence of layer sliding. Therefore, possibly to accommodate the pressure compression behavior, the layers slide and it gives an impression of increased volume.

Each unit cell contains four octahedra, two of them originated from Re5 atoms (type I) and other two from Re6 atoms (type II) surrounded by sulfur atoms. Evolution of octahedral volume (OV) and distortion index (DI) of both type octahedra of $1 \mathrm{~T}^{\prime}$ with pressure are shown in Fig. S6 in the supplementary material. In Table I, $\mathrm{OV}$, average bond length $(\mathrm{ABL})$, and $\mathrm{DI}$ in few pressure points are presented. Their values in the ambient sample are found to be very similar irrespective of the types (I and II) of the octahedra. In the $1 \mathrm{~T}^{\prime}$ structure at $6.1 \mathrm{GPa}$, the $\mathrm{OV}$ of the different types are observed to differ by $0.2 \AA^{3}$ and ABL by only $0.006 \AA$. But, a large difference 0.018 in the DI of different types of octahedra with respect to the value 0.0008 in the ambient structure is observed. DI is defined as $\frac{1}{n} \sum_{i=1}^{n} \frac{d_{i}-d_{a v}}{d_{a v}}$, where $d_{i}$ is the distance from the central rhenium atom to the $i$ th coordinating atom and $d_{a v}$ is the ABL. ${ }^{48}$ High value in the DI in type II octahedral can be noted in Table I, which means it is highly distorted. OV of type II increases slowly from $19.3234 \AA^{3}$ to a value $19.7951 \AA^{3}$ at $14.7 \mathrm{GPa}$, while it decreases rapidly (from 19.0949 to $15.892 \AA^{3}$ in the pressure range of 6.1-14.7 GPa) in the case of type I octahedra. DIs are observed to be highly sensitive to pressure and reached to a maximum value at 14.7 GPa indicating highly distorted octahedra. Both the OVs are observed to be increased by a maximum $6.9 \%$ in the pressure range of $16-23 \mathrm{GPa}$, while in the same pressure range, the unit cell volume is increased by $3 \%$ as evident from Fig. 4. Interestingly, $20 \%-25 \%$ decrease in the DIs for both types of octahedra in the above pressure range are observed. From Fig. 5(b), one can see S3 atom is shared by both types of octahedra in the $1 \mathrm{~T}^{\prime}$ phase, and it is also evident from Table I that ABLs are increased by $2 \%$ at 
(a)

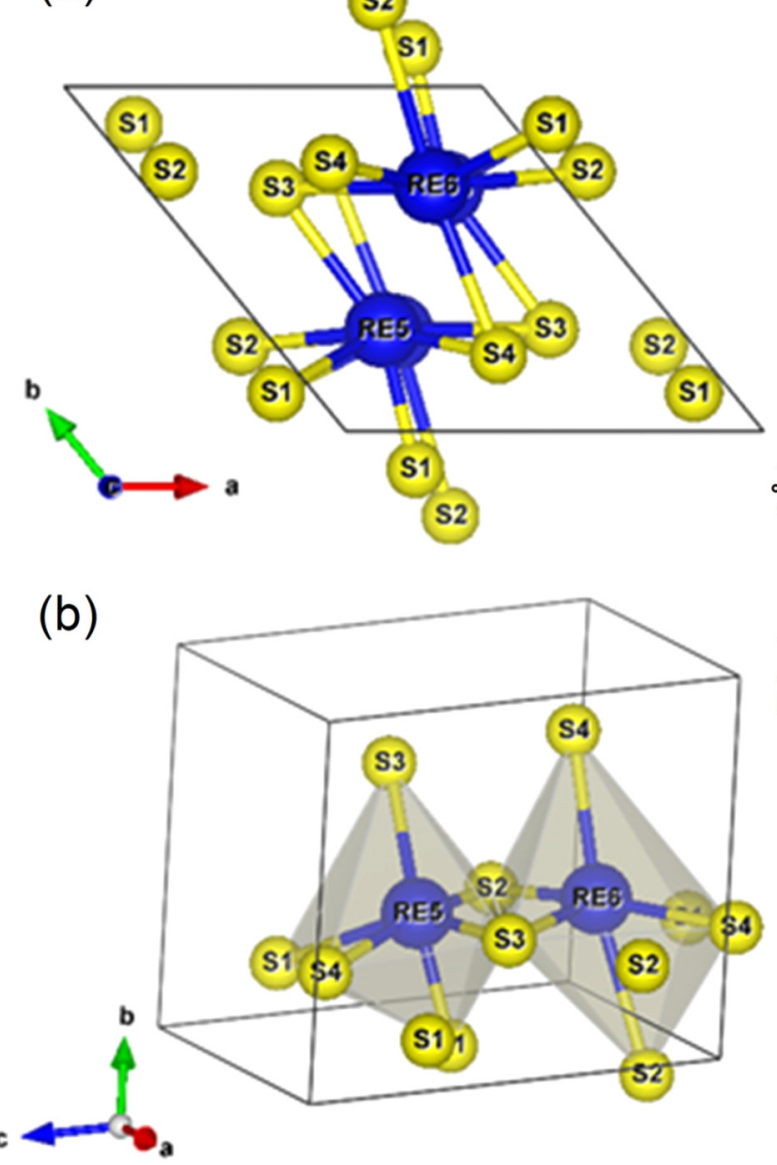

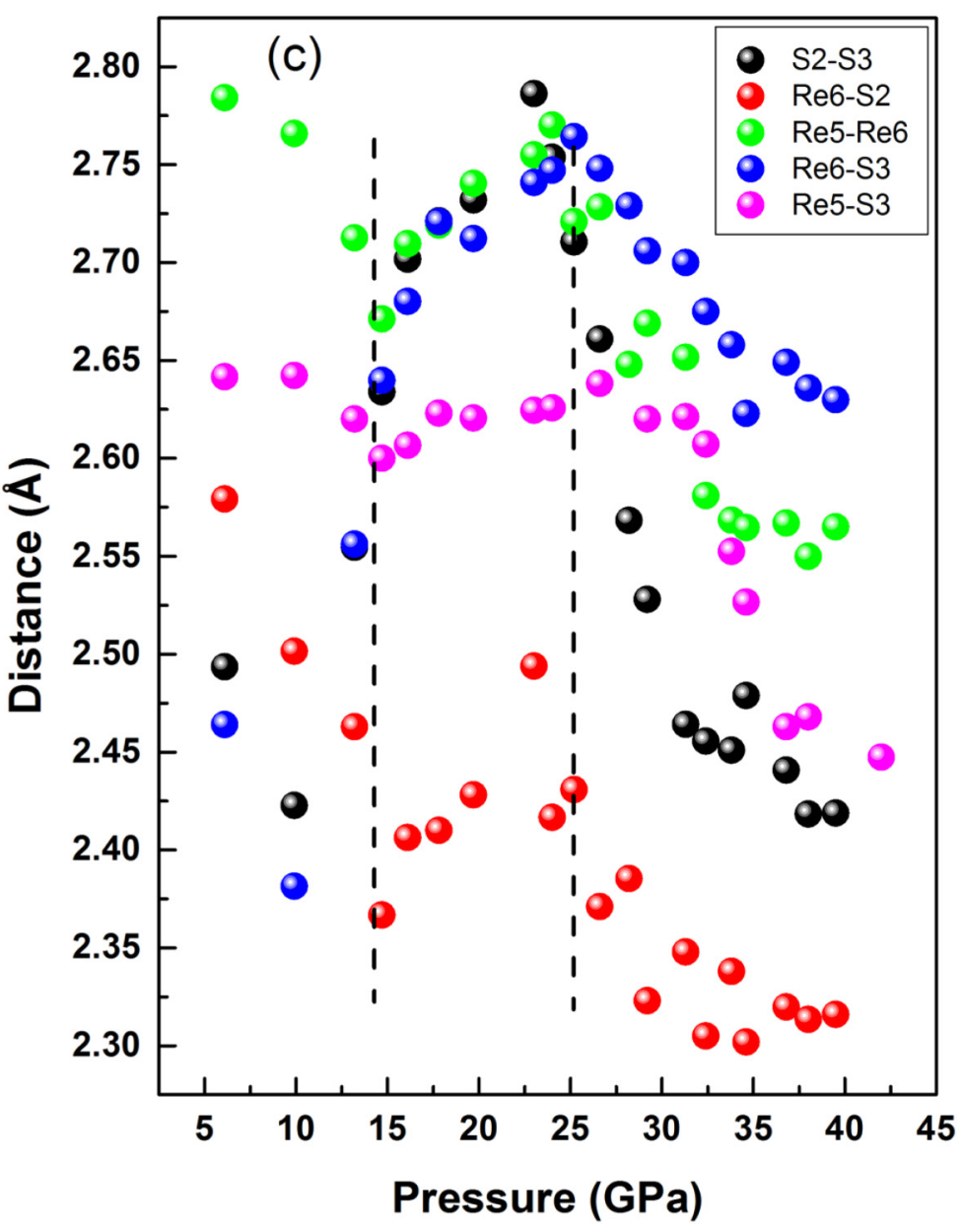

FIG. 5. (a) Vesta plot of the unit cell of the distorted $1 T^{\prime}$-phase in the ab-plane. (b) Two types of octahedra sharing S3 atom. (c) Evolution of atom distances as a function of pressure. Vertical dotted lines represent the separations between region-I and the volume expansion region, and the volume expansion region and region-II.

TABLE I. The derived parameters from the analysis of the XRD patters using $\mathrm{GSAS}^{33}$ at a few pressure points.

\begin{tabular}{|c|c|c|c|c|}
\hline Pressure $(\mathrm{GPa})$ and structure & Type of octahedra & $\mathrm{OV}\left(\AA^{3}\right)$ & $\mathrm{ABL}(\AA ̊)$ & DI \\
\hline \multirow[t]{2}{*}{ Ambient, 1T } & Type I & 17.8390 & 2.4293 & 0.03770 \\
\hline & Type II & 17.9318 & 2.4235 & 0.03688 \\
\hline \multirow[t]{2}{*}{$6.1,1 \mathrm{~T}^{\prime}$} & Type I & 19.0949 & 2.5305 & 0.02874 \\
\hline & Type II & 19.3234 & 2.5241 & 0.04683 \\
\hline \multirow[t]{2}{*}{$14.7,1 \mathrm{~T}^{\prime}$} & Type I & 15.8920 & 2.4095 & 0.07568 \\
\hline & Type II & 19.7951 & 2.5428 & 0.05651 \\
\hline \multirow[t]{2}{*}{$23,1 \mathrm{~T}^{\prime}$} & Type I & 16.5819 & 2.4511 & 0.05077 \\
\hline & Type II & 21.1620 & 2.6020 & 0.04217 \\
\hline \multirow[t]{2}{*}{$31.3,1 \mathrm{~T}^{\prime}$} & Type I & 15.7427 & 2.3966 & 0.08317 \\
\hline & Type II & 19.1420 & 2.5110 & 0.05340 \\
\hline
\end{tabular}


$23 \mathrm{GPa}$ with respect to $14.7 \mathrm{GPa}$. Therefore, one can expect, increase in Re5-S3 or Re6-S3 distance should decrease S2-S3 distance. In contrary, we observed an increase in the S2-S3 distance at above pressure region. So for the stabilization of the $1 \mathrm{~T}^{\prime}$ phase, a minimization in DIs is observed due to increase in the unit cell volume, which can be accommodated by the layer sliding. In layered metalorganic framework, layer sliding was reported to be responsible for the volume expansion. ${ }^{41}$ Above $23 \mathrm{GPa}$, a systematic behavior like decrease in the octahedra volume, average bond length, and pressure induced increase in the DI values are observed.

As a complementary study to XRD measurements, we have carried out a Raman spectroscopy investigation up to about $44 \mathrm{GPa}$ on exfoliated $\mathrm{ReS}_{2}$. Earlier high pressure Raman investigation on single crystal $\mathrm{ReS}_{2}$ using a methanol-ethanol mixture as pressure transmitting medium and a $532 \mathrm{~nm}$ laser excitation source did report the following: (i) emergence of few new modes in the pressure range of $8-19.6 \mathrm{GPa}$ and (ii) changes in the Raman mode behavior: change in the slope of intensity ratio of $E_{g}-3$ and $E_{g}-$ 4 modes. ${ }^{17}$ These were attributed to a phase transition starting at 8 $\mathrm{GPa}$ and completing at $19.6 \mathrm{GPa} .{ }^{17}$ But other Raman measurements on the powder samples do not report any of the above anomalous behavior, even though evidence of structural transition is observed in their studies. ${ }^{718}$ In Fig. 6(a), we have shown the Raman spectra of $\mathrm{ReS}_{2}$ from ambient to a pressure $7.7 \mathrm{GPa}$ in the frequency range of $250-550 \mathrm{~cm}^{-1}$. These modes originate from the vibration of sulfur atoms. All the $C_{p}$ Raman modes corresponding to the coupled vibration of rhenium and sulfur atoms are found to decrease in intensity with pressure and finally become undetectable above $6.3 \mathrm{GPa}$. In the same pressure range, a few new diffraction peaks are observed and the pattern is indexed to a different set of lattice parameters showing an isostructural transition to the distorted $1 \mathrm{~T}^{\prime}$ phase. Therefore, disappearance of $C_{p}$ Raman modes may be related to the increased distortion in the sample, which increases the disorder in Re-S coupling parameters. Vibrational modes $E_{g}-6$ and $A_{1 g}-4$ of the sulfur atoms are found to survive in the whole pressure range in our study. Raman spectra in the frequency range of $120-265 \mathrm{~cm}^{-1}$ corresponding to the vibration of the rhenium atoms at the selected pressure points are shown in Fig. 6(b). Above $6.3 \mathrm{GPa}$, few Raman modes are found to emerge with pressure. We have indicated the
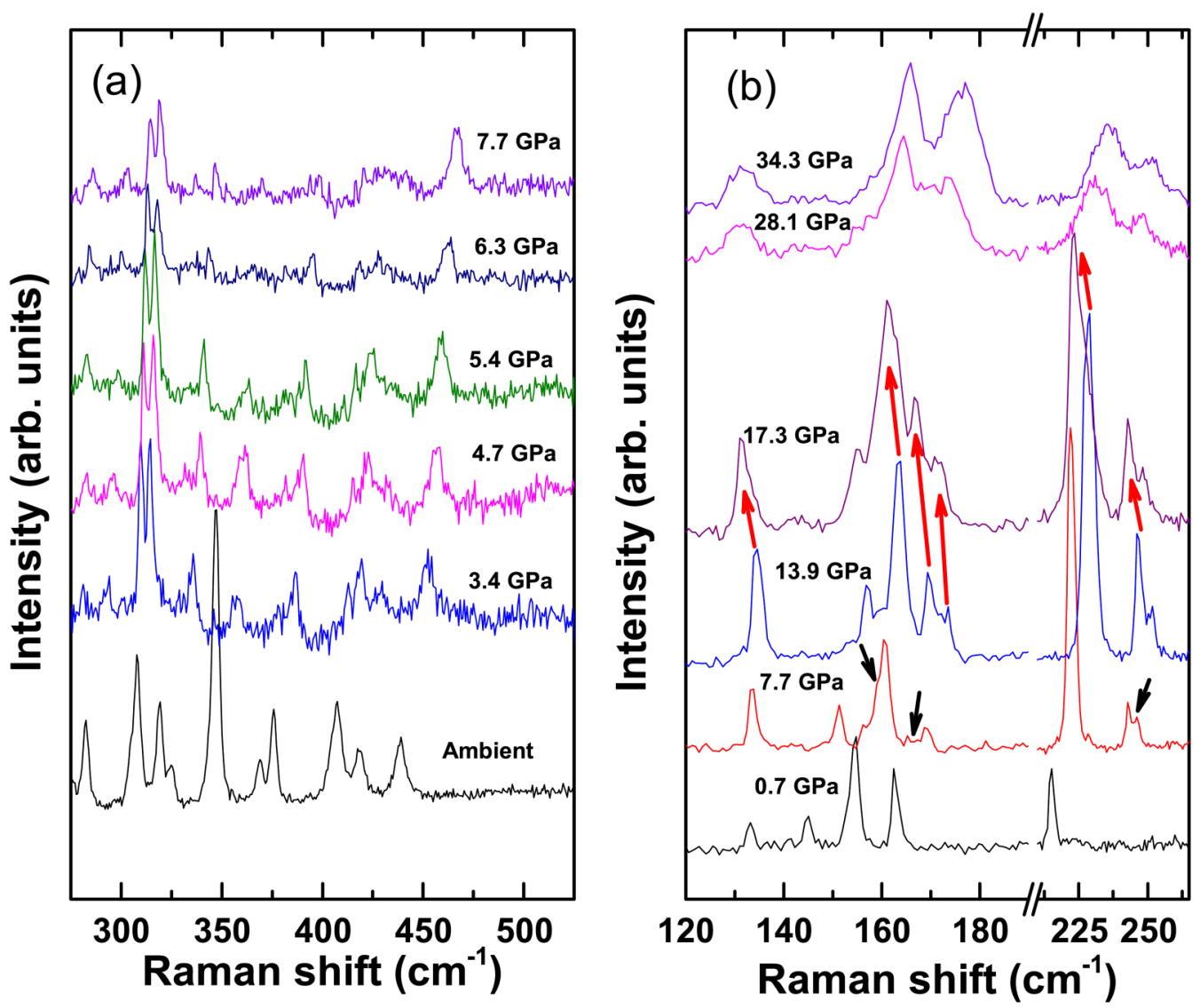

FIG. 6. (a) Evolution of Raman spectrum in the low pressure range of $0-7.7 \mathrm{GPa}$ from 250 to $550 \mathrm{~cm}^{-1}$ at selected pressure points. (b) Evolution of Raman spectrum at the pressure range of $0.7-34.3 \mathrm{GPa}$ from 120 to $265 \mathrm{~cm}^{-1}$ at selected pressure points. Black arrows represent new modes, while red arrows show the mode softening. 

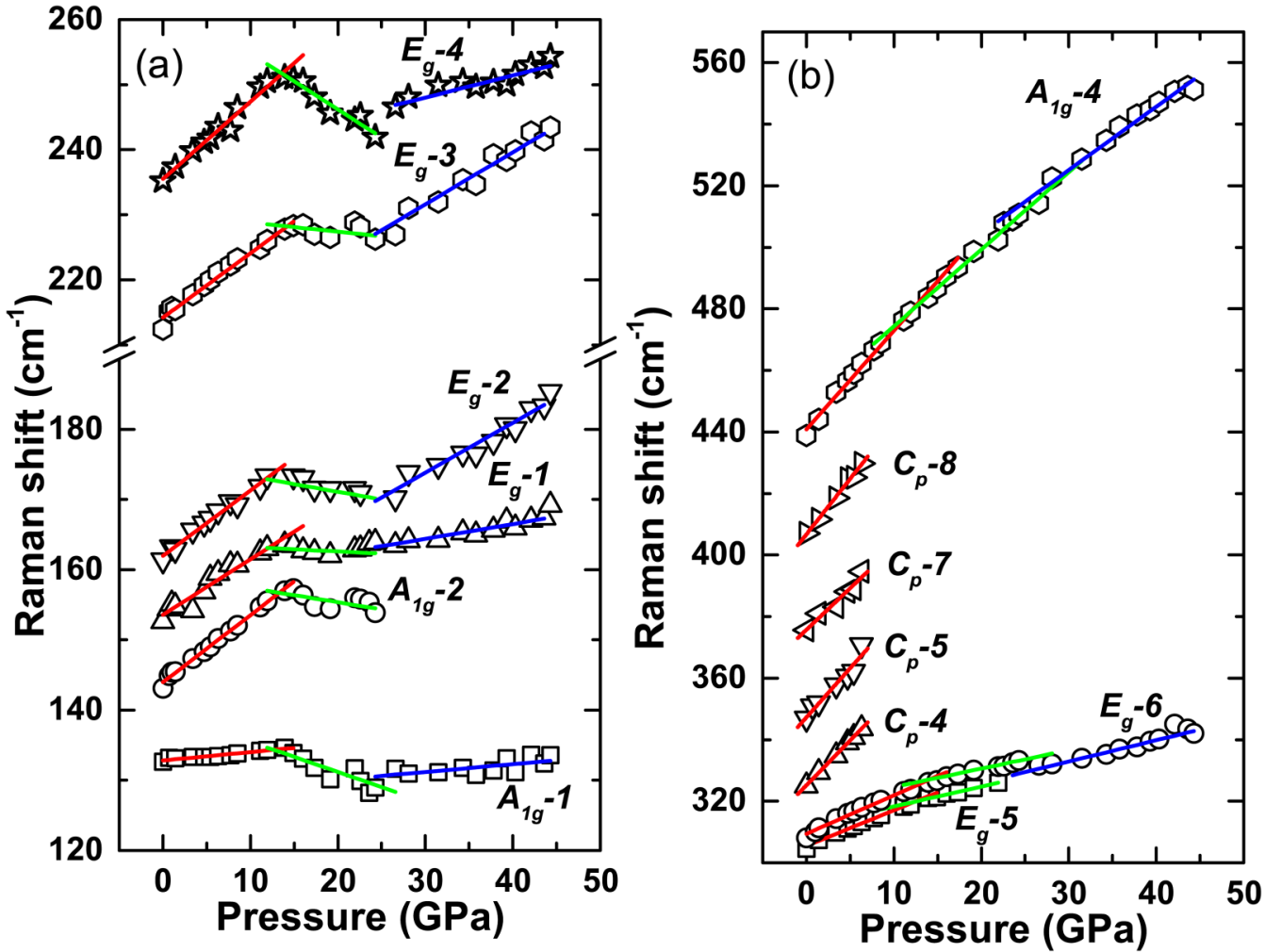

FIG. 7. (a) Pressure evolution of Raman modes corresponding to the rhenium atom vibrations. (b) Raman modes corresponding to the sulfur atom vibrations with pressure. Red, green, and blue lines represent linear fitting to the data in the pressure range of 0-14 (1st range), 15-25 (2nd range), and 25-45 GPa (3rd range), respectively.

new modes by black arrows at $7.7 \mathrm{GPa}$ pressure spectrum in Fig. 6(b). The emergence of these Raman modes itself is an evidence of phase transition. The intensity of these new modes are found to increase with pressure. New Raman modes are found to merge with the nearest existing modes and broaden extensively above $24 \mathrm{GPa}$. From Fig. 6(b), it is evident that Raman modes are red shifted at 17.3 GPa with respect to $13.9 \mathrm{GPa}$ data, and these are marked by the red arrows.

To have more insight, we have plotted pressure evolution of Raman modes corresponding to rhenium atom vibrations in Fig. 7 (a) and those related to sulfur atoms vibrations in Fig. 7(b). Interestingly, three different linear ranges (1st range: 0-14 GPa, 2nd range: $15-25 \mathrm{GPa}$, and 3rd range: $25-45 \mathrm{GPa}$ ) are noted in Fig. 7. 1 st range belongs to the $1 \mathrm{~T}$-phase and region-I of the $1 \mathrm{~T}^{\prime}$-phase, and it is observed that both of them have similar bulk modulus values. 2nd range is from 15 to $25 \mathrm{GPa}$, where volume expansion takes place. $3 \mathrm{rd}$ range belongs to region-II of the $1 \mathrm{~T}^{\prime}$-phase. Raman mode values vs pressure show change in the slope at around $14 \mathrm{GPa}$ and $25 \mathrm{GPa}$. In the 1st range of the pressure $0-14 \mathrm{GPa}$, the modes are found to be highly sensitive to pressure. It is in agreement with the $\mathrm{x}$-ray studies, which show high compressibility of the sample in the pressure range of $0-16 \mathrm{GPa}$. Above $15 \mathrm{GPa}$, in the 2 nd range, all the modes soften with pressure up to $25 \mathrm{GPa}$, which gives rise to the negative slopes. One possible explanation of the softening of the mode values with pressure can be attributed to the decrease in bond strength, which is caused by the volume expansion induced by the layer sliding as observed in our x-ray diffraction studies. Above $18 \mathrm{GPa}$, the out-of-plane mode $A_{1 g}-2$ is broaden extensively and disappear above $25 \mathrm{GPa}$. All remaining modes are found to harden with pressure above $25 \mathrm{GPa}$ (in the 3rd range of Raman study/region-II of $1 \mathrm{~T}^{\prime}$ in $\mathrm{x}$-ray measurements) at a slow rate with respect to the 1st range. The smaller sensitivity of the Raman mode shift with pressure reveal the low compressibility of the sample above $25 \mathrm{GPa}$, which supports $\mathrm{x}$-ray diffraction studies of this work. First-principles study of $\mathrm{ReS}_{2}$ under pressure show pressure hardening of the Raman modes with smaller slope in the $1 \mathrm{~T}^{\prime}$ phase with respect to those of the $1 \mathrm{~T}$ phase. ${ }^{19}$ Though no softening of any Raman mode is observed corresponding to the sulfur-atom vibrational modes, $E_{g}-6$ and $A_{1 g}-4$ modes show three different linear regions distinguished by three different slope values. The out-of-plane vibrational mode, $A_{1 g}-4$, of the sulfur atom is found to be highly sensitive to pressure in the 1st range of pressure in the Raman measurements with respect to all the other modes. Modes related to rhenium-sulfur atom coupled vibrations, $C_{p} \mathrm{~s}$ are also found to be highly sensitive to pressure but disappeared above $6.3 \mathrm{GPa}$. $E_{g}-5$ mode broadens and disappears above $23 \mathrm{GPa}$. Slopes of the pressure variation of Raman modes are listed in Table II in the supplementary material. 
Softening in the Raman modes in this study are observed just above the freezing point $(\sim 11 \mathrm{GPa})$ of methanol-ethanol mixture. ${ }^{49}$ For the exact confirmation of the Raman mode behavior, we have carried out Raman spectroscopy measurements using three different types of pressure transmitting media. In Fig. S7 in the supplementary material, we have compared the mode evolution with pressure for a few selected pressure points in the pressure range of 14.8-26.6 GPa. From this figure, it is evident that the softening of modes in the pressure range of 15-24 GPa are observed in experiments using different pressure transmitting media: Fig. S7(a) in the supplementary material corresponds to ethanol-methanol mixture; Fig. S7(b) in the supplementary material corresponds to iso-propanol; and Fig. S7(c) in the supplementary material corresponds to silicone oil. A larger broadening of Raman modes are observed in Fig. S7(c) in the supplementary material compared to other figures and can be related to non-hydrostatic stress induced by silicone oil as it solidifies at much lower pressure. Similar softening behavior even with the use of different pressure media confirms that the mode softening is related to sample response to pressure only. The phonon softening can be related to anomalous volume expansion observed in the sample using neon as pressure transmitting medium. Theoretical thermodynamic calculations by Sheremetyeva et al. show that the $1 \mathrm{~T}^{\prime}$ phase is more favorable in the ambient condition with respect to the $1 \mathrm{~T}$ phase. ${ }^{19}$ But the synthesis procedure of these single crystal materials support the stability of the $1 \mathrm{~T}$ phase due to unavoidable finite strain. It can be noted that the Eulerian strain value increases rapidly up to $16 \mathrm{GPa}$ to a value 0.075 , then it slowly decreases to 0.06 at $25 \mathrm{GPa}$, as evident from Fig. 4(b). It can be seen in Fig. 7(a) that the maximum softening in the mode values is observed in $E_{g}-4$, which relates to the in-plane vibration of the rhenium atom. Therefore, one can speculate that growing strain starts decreasing due to the expansion in the Re5-Re6 distance, lattice parameter, and volume, which affect the in-plane lattice vibrations mostly. So, pressure induced strain has an important impact in the stabilization of the structure of $\mathrm{ReS}_{2}$ similar to other exfoliated TMDs. ${ }^{25,26}$ More theoretical and experimental investigation are required to understand the exact effect of pressure induced strain on these systems.

\section{CONCLUSIONS}

In the present study, we have carried out detailed high pressure Raman and XRD investigations on an exfoliated $\mathrm{ReS}_{2}$ sample. The ambient sample is found to have a distorted $1 \mathrm{~T}$ structure. A structural transition to the distorted $1 \mathrm{~T}^{\prime}$ phase is detected from the emergence of a few Bragg peaks above $6 \mathrm{GPa}$. Lattice expansion due to the decrease in the Eulerian strain is observed in the pressure range of 16-25 GPa, where all the Raman modes corresponding to rhenium atom vibrations show mode softening irrespective of the pressure transmitting medium. These observations show instability in the new structure in the above pressure range and show systematic behavior with pressure above $25 \mathrm{GPa}$. The volume expansion in the intermediate pressure range can be related to layer sliding to minimize the lattice strain.

\section{SUPPLEMENTARY MATERIAL}

See the supplementary material for additional tables and figures related to high pressure structure and Raman data of $\operatorname{ReS}_{2}$.

\section{AUTHORS' CONTRIBUTIONS}

All authors contributed equally to this manuscript. All authors reviewed the final manuscript.

\section{ACKNOWLEDGMENTS}

G.D.M. wishes to thank the Ministry of Earth Sciences, Government of India for financial support under Project Grant No. MoES/16/25/10-RDEAS. P.S. and B.G. wish to thank the DST, INSPIRE program by the Department of Science and Technology, Government of India for financial support. G.D.M. and P.S. thankfully acknowledge the DST-DESY project under Department of Science and Technology, Government of India for financial support to carry out the proposed experiment at DESY, Germany.

The authors declare no competing financial interests.

\section{DATA AVAILABILITY}

The data that support the findings of this study are available from the corresponding author upon reasonable request.

\section{REFERENCES}

${ }^{1}$ Y. Li, Z. Zhou, S. Zhang, and Z. Chen, J. Am. Chem. Soc. 130, 16739 (2008).

${ }^{2}$ Q. H. Wang, K. Kalantar-Zaden, A. Kis, J. N. Coleman, and M. S. Strano, Nat. Nanotechnol. 7, 699 (2012).

${ }^{3}$ Z. H. Chi, X. M. Zhao, H. Zhang, A. F. Goncharov, S. S. Lobanov, T. Kagayama, M. Sakata, and X. J. Chen, Phys. Rev. Lett. 113, 036802 (2014).

${ }^{4}$ M. S. Choi, D. Qu, D. Lee, X. Liu, K. Watanabe, T. Taniguchi, and W. J. Yoo, ACS Nano 8, 9332 (2014).

${ }^{\mathbf{5}}$ N. Huo, J. Kang, Z. Wei, S. Li, J. Li, and S. Wei, Adv. Funct. Mater. 24, 7025 (2014).

${ }^{6}$ D. Akinwande, C. J. Brennan, J. S. Bunch, P. Egberts, J. R. Felts, H. Gao, R. Huang, J.-S. Kim, T. Li, Y. Li, K. M. Liechti, N. Lu, H. S. Park, E. J. Reed, P. Wang, B. I. Yakobson, T. Zhang, Y.-W. Zhang, Y. Zhou, and Y. Zhu, Extreme Mech. Lett. 13, 42 (2017).

${ }^{7}$ S. Tongay, H. Sahin, C. Ko, A. Luce, W. Fan, K. Liu, J. Zhou, Y.-S. Huang, C.-H. Ho, J. Yan, D. F. Ogletree, S. Aloni, J. Ji, S. Li, J. Li, F. M. Peeters, and J. Wu, Nat. Commun. 5, 3252 (2014).

${ }^{8}$ Z. Chi, X. Chen, F. Yen, F. Peng, Y. Zhou, J. Zhu, Y. Zhang, X. Liu, C. Lin, S. Chu, Y. Li, J. Zhao, T. Kagayama, Y. Ma, and Z. Yang, Phys. Rev. Lett. 120, 037002 (2018).

${ }^{\mathbf{9}}$ M. Calandra and F. Mauri, Phys. Rev. Lett. 106, 196406 (2011).

${ }^{10}$ U. Chatterjee, J. Zhao, M. Iavarone, R. di Capua, J. P. Castellan, G. Karapetrov, C. D. malliakas, M. G. Kanatzidis, H. Claus, J. P. C. Ruff, F. Weber, J. van Wezel, J. C. Kampuzano, R. Osborn, M. Randeria, N. Trivedi, M. R. Norman, and S. Rosenkranz, Nat. Commun. 6, 6313 (2015).

${ }^{11}$ M. S. Silverma, Inorg. Chem. 6, 1063 (1967).

${ }^{12}$ H. Katzke, P. Toledano, and W. Depmeier, Phys. Rev. B 69, 134111 (2004).

${ }^{13}$ A. N. Enyashin, L. Yadgarov, L. Houben, I. Popov, M. Weidenbach, R. Tenne, M. B. Sadan, and G. Seifert, J. Phys. Chem. C 115, 24586 (2011).

${ }^{14}$ L. Hromadova, R. Martonak, and E. Tosatti, Phys. Rev. B 87, 144105 (2013).

${ }^{15}$ E. Liu, Y. Fu, Y. Wang, Y. Feng, H. Liu, X. Wan, W. Zhou, B. Wang, L. Shao, C.-H. Ho, Y.-S. Huang, Z. Cao, L. Wang, A. Li, J. Zeng, F. Song, X. Wang, Y. Shi, H. Yuan, H. Y. Hwang, Y. Cui, F. Miao, and D. Xing, Nat. Commun. 6, 6991 (2015).

${ }^{16}$ Y. Feng, W. Zhou, Y. Wang, J. Zhou, E. Liu, Y. Fu, Z. Ni, X. Wu, H. Yuan, F. Miao, B. Wang, X. Wan, and D. Xing, Phys. Rev. B. 92, 054110 (2015).

${ }^{17}$ Y. Yan, C. Jin, J. Wang, T. Qin, F. Li, K. Wang, Y. Han, and C. Gao, J. Phys. Chem. Lett. 8, 3648 (2017).

${ }^{18}$ P. Wang, Y. Wang, J. Qu, Q. Zhu, W. Yang, J. Zhu, L. Wang, W. Zhang, D. He, and Y. Zhao, Phys. Rev. B 97, 235202 (2018). 
${ }^{19} \mathrm{~N}$. Sheremetyeva, D. Tristant, A. Yoshimura, J. Gray, L. Liang, and V. Meunier, Phys. Rev. B 100, 214101 (2019).

${ }^{20}$ R. Oliva, M. Laurien, F. Dybala, J. Kopaczek, Y. Qin, S. Tongay, O. Rubel, and R. Kudrawiec, npj 2D Mater. Appl. 3, 20 (2019).

${ }^{21}$ A. P. Nayak, S. Bhattacharyya, J. Zhu, J. Liu, X. Wu, T. Pandey, C. Jin, A. K. Singh, D. Akinwande, and J. F. Lin, Nat. Commun. 5, 3731 (2014).

${ }^{22}$ A. P. Nayak, Z. Yuan, B. Cao, J. Liu, J. Wu, S. T. Moran, T. Li, D. Akinwande, C. Jin, and J. F. Lin, ACS Nano 9, 9117 (2015).

${ }^{23}$ Z. Zhao, H. Zhang, H. Yuan, S. Wang, Y. Lin, Q. Zeng, G. Xu, Z. Liu, G. K. Solanki, K. D. Patel, Y. Cui, H. Y. Hwang, and W. L. Mao, Nat. Commun. 6, 7312 (2015).

${ }^{24}$ S. Duwal and C. S. Yoo, J. Phys. Chem. C 120, 5101 (2016).

${ }^{25}$ P. Saha, B. Ghosh, R. Jana, and G. D. Mukherjee, J. Appl. Phys. 123, 204306 (2018).

${ }^{\mathbf{2 6}}$ P. Saha, B. Ghosh, A. Mazumder, and G. D. Mukherjee, Mater. Res. Express 7, $2(2020)$.

${ }^{27}$ D. Hou, Y. Ma, J. Du, J. Yan, C. Ji, and H. Zhu, J. Phys. Chem. Solids 71, 1571 (2010).

${ }^{28}$ D. Zhou, Y. Zhou, C. Pu, X. Chen, P. Lu, X. Wang, C. An, Y. Zhou, F. Miao, C. H. Ho, J. Sun, Z. Yang, and D. Xing, npj Quantum Mater. 2, 19 (2017).

${ }^{\mathbf{2 9}}$ H. K. Mao, J. Xu, and P. M. Bell, J. Geophys. Res. 91, 4673, (1986).

${ }^{30}$ A. Dewaele, M. Torrent, P. Loubeyre, and M. Mezouar, Phys. Rev. B 78, 104102 (2008).

${ }^{31}$ C. Prescher and V. B. Prakapenka, High Pressure Res. 35, 223 (2015).

${ }^{32} \mathrm{R}$. Shirley, The CRYSFIRE 2002 System for Automatic Powder Indexing: Users Manual (The Lattice Press, Guildford, 2002).

${ }^{33}$ B. H. Toby, J. Appl. Crystallogr. 34, 210 (2001).

${ }^{34}$ H. H. Murray, S. P. Kelty, R. R. Chianelli, and C. S. Day, Inorg. Chem. 33, 4418 (1994).
${ }^{35}$ H. J. Lamfers, A. Meetsma, G. A. Wiegers, and J. L. de Boer, J. Alloys Compd. 241, 34 (1996).

${ }^{36}$ S. Zhang, N. Mao, N. Zhang, J. Wu, L. Tong, and J. Zhang, ACS Nano 11, 10366 (2017).

${ }^{37}$ D. A. Chenet, O. B. Aslan, P. Y. Huang, C. Fan, A. M. Van Der Zande, T. F. Heinz, and J. C. Hone, Nano Lett. 15, 5667 (2015).

${ }^{38}$ X.-F. Qiao, J.-B. Wu, L. Zhou, J. Qiao, W. Shi, T. Chen, X. Zhang, J. Zhang, W. Li, and P.-H. Tan, Nanoscale 8, 8324 (2016).

${ }^{39}$ Y. Yan, H. Liu, Y. Han, F. Li, and C. Gao, Phys. Chem. Chem. Phys. 20, 24927 (2018).

${ }^{40}$ A. McCreary, J. R. Simpson, Y. Wang, D. Rhodes, K. Fujisawa, L. Balicas, M. Dubey, V. H. Crespi, M. Terrones, and A. R. Hight Walker, Nano Lett. 17, 5897 (2017).

${ }^{41}$ Q. Zeng, K. Wang, Y. Qiao, X. Li, and B. Zou, J. Phys. Chem. Lett. 8, 1436 (2017).

${ }^{42}$ A. Polian, M. Gauthier, S. M. Souza, D. M. Triches, J. CardosodeLima, and T. A. Grandi, Phys. Rev. B 83, 113106 (2011).

${ }^{43}$ R. Jana, P. Saha, V. Pareek, A. Basu, S. Kapri, S. Bhattacharyya, and G. D. Mukherjee, Sci. Rep. 6, 31610 (2016).

${ }^{44}$ P. Saha, K. Glazyrin, and G. D. Mukherjee, arXiv:1905.11030 (2019).

${ }^{45}$ R. J. Angel, "High-pressure, high-temperature crystal chemistry," in Reviews in Mineralogy \& Geochemistry, edited by R. M. Hazen and R. T. Downs (CRC Press, Boka Raton, FL, 2001), Vol. 41, p. 35.

${ }^{46}$ F. D. Murnaghan, Am. J. Math. 59, 235 (1937).

${ }^{47}$ F. Birch, Phys. Rev. B 71, 809 (1947).

${ }^{48}$ W. H. Baur, Acta Crystallogr. B 30, 1195 (1974).

${ }^{49}$ S. Klotz, J.-C. Chervin, P. Munsch, and G. L. Marchandh, J. Phys. D Appl. Phys. 42, 075413 (2009). 